\title{
Virtual Multichannel SAR for Ground Moving Targets Imaging
}

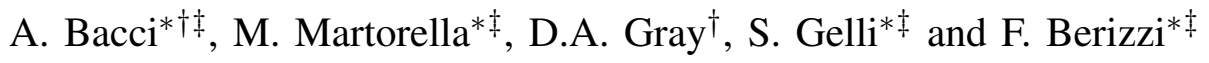 \\ *Department of Information Engineering, University of Pisa, Italy \\ Email: alessio.bacci@for.unipi.it, [marco.martorella, fabrizio.berizzi] @iet.unipi.it \\ †University of Adelaide Radar Research Centre, EEE School, Australia \\ Email: dgray@eleceng.adelaide.edu.au \\ $\ddagger$ CNIT Radar and Surveillance System (RaSS) National Laboratory, Pisa, Italy
}

\begin{abstract}
Slow moving ground targets are invisible within SAR images since they appear defocused and their backscattered signal completely overlap the focused ground return. In order for this targets to be detected and refocused the availability of some spatial degrees of freedom is required. This allows for space/slow time processing to be applied to mitigate the ground clutter. However, Multichannel SAR (M-SAR) systems are very expensive and the requirements in terms of baseline length can be very restrictive. In this paper a processing scheme that exploits high PRF single channel SAR system to emulate a multichannel SAR is presented. The signal model for both target and clutter components are presented and the difference with respect to an actual M-SAR are highlighted. The effectiveness of the proposed processing is then demonstrated on simulated a measured dataset.
\end{abstract}

\section{INTRODUCTION}

Synthetic Aperture Radar (SAR) systems exploit the radar motion to provide high resolution electromagnetic images of an illuminated scene [8] and have been used for decades for earth observation applications. Since the modern SAR systems can provide very detailed electromagnetic images with low revisiting time they can be used in homeland security applications. In this kind of scenario the capability of obtaining high resolution images of non-cooperative moving targets becomes fundamental.

It is well known that moving targets within the illuminating area appear defocused and, especially in the case of slow moving targets, their detection and imaging are challenging tasks [25]. This is due to 
the fact that the return of a slow moving target overlaps with that of the ground clutter that is much stronger making the targets of interest invisible.

A solution to the imaging of moving target issue is proposed in [22],[10] in which ISAR processing is successfully applied to targets detected within SAR images. In that paper only maritime targets are considered since in that case detection is quite straightforward.

Conversely, in the case of slowly moving ground targets different detection techniques can be found in literature. The earliest method for discriminating moving target signals from static scene return in single channel SAR systems is based on the differences in the Doppler spectra of stationary ground clutter and the return of moving targets [12], [25]. This technique is based on the assumption that the radar PRF is high enough to obtain a region in the Doppler frequency domain that is free of the static scene components. Whilst the technique can be readily applied to single channel data, it suffers from some shortcomings. First of all, it requires a high PRF that results in both a reduction in the SAR swath width and an increase in the size of the block of data to be processed. Secondly, it generally fails when dealing with the detection of slow moving targets for which Doppler frequency falls completely within the Doppler bandwidth of the static scene. Other techniques for detecting moving targets with single channel SAR systems are based on change detection algorithms which make use of different looks of the same scene at different times [31], or by the application of non-uniform sampling, i.e., non-uniform Pulse Repetition Intervals (PRIs) [20]. However, the effectiveness of all these methods when dealing with slow moving targets is limited in principle because the moving target returns cannot be separated from the static scene return in the Doppler domain.

When spatial Degrees of Freedom (DoFs) are available more powerful methodologies can be applied. These techniques exploit the capability of collecting multiple spatial samples, i.e., echoes received from different antenna elements, and multiple time samples, i.e., echoes collected at different PRI intervals to mitigate strong ground clutter interference. Along Track Interferometry (ATI) [24][17][9], Displaced Phase Centre Array (DPCA) [13][29], Time-Frequency Transforms (TFT) [11][6][21] and Space Time Adaptive processing (STAP) are examples of the use of multichannel SAR systems for mitigating the deleterious effects of clutter. Specifically, STAP techniques are widely used for clutter mitigation purposes and have been widely treated for general airborne radar system in the case of slowly moving ground targets [19] [18] [30].

In recent years, with the development of SAR systems with multichannel capability (M-SAR), the application of Space Time Adaptive Processing to imaging systems has gained the attention of the radar 
scientific community. In [14], [15] optimum space-time processing for moving target detection in SAR was analytically derived, the implementation of slow-time STAP in both time and frequency domain was considered and a comparison between the optimal solution and a number of reduced rank methods carried out. The slow-time and frequency approaches are known as pre- and post-Doppler STAP respectively and in [28], [26] and [27] STAP processing of SAR data for jammer suppression was widely treated by considering both slow and fast time approaches. Whilst STAP and its derivative approaches have been extensively investigated for suppressing clutter for catching invisible slow moving ground targets, its use for both clutter suppression and imaging of moving targets have only recently been considered. In [2] a combination of STAP and ISAR techniques for the imaging of moving targets in SAR images has been proposed. The approach in [2] has been formulated in the space/slow-time domain and it can, as observed in [1], readily be extended the space Doppler domain [5].

In this paper a technique that makes SDAP (Space Doppler Adaptive Processing) [5] applicable to single channel SAR systems with high PRF by emulating a virtual M-SAR is presented. The approach is very similar to the one presented in [16], in which the information acquired with a multichannel system are exploited to synthesize a single channel SAR system with higher PRF with respect to the actual one. Conversely, the approach suggested in our work exploits high PRF SAR to emulate a multichannel SAR system making virtual spatial DoFs available at the price of a reduction of the non-ambiguous Doppler region.

It is worth highlighting that, a high PRF causes some issues in the radar design since it leads to a reduction of the non-ambiguous range and an increase of the amount of data to be acquired and processed. However, the synthesis of a virtual M-SAR allows for avoiding the use of multiple receiving chains and all the issues related to cross-channel calibration. Moreover, as it will be explained in the following, the baseline and length of the virtual array can be imposed by suitably choosing the PRF and the platform velocity without taking into account the physical size of the antennas.

This paper is organised as follows: the signal model for both the target and the clutter components is presented in Sect. II and the difference between an actual and a virtual M-SAR are explained as well as the constraints in the system design. A review of the SDAP-ISAR processing is performed in Sect.III. Results obtained on a simulated dataset are shown in Sect. IV. The controlled environment allows for a deep analysis of the peculiarities of the SDAP processing applied to virtual M-SAR and the shortcomings with respect to the use of an actual M-SAR. Results obtained by processing a measured dataset are shown in Sect.V. In particular a comparison with the results obtained by processing an actual two channels SAR data [5] is provided. These results proves the effectiveness of the proposed processing. 


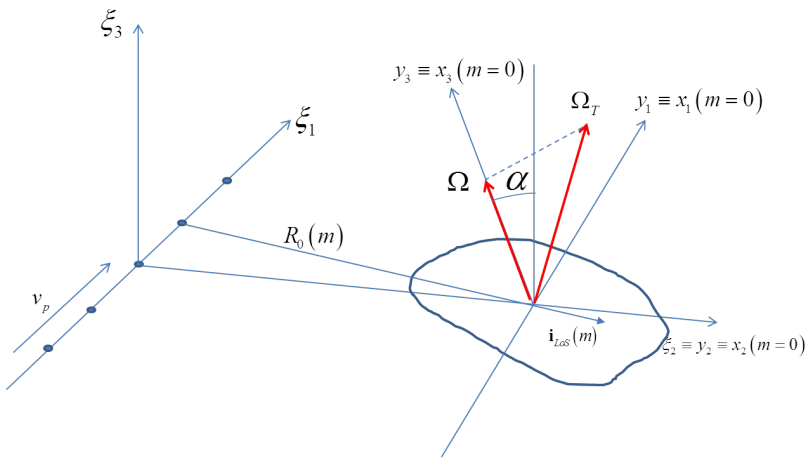

(a)

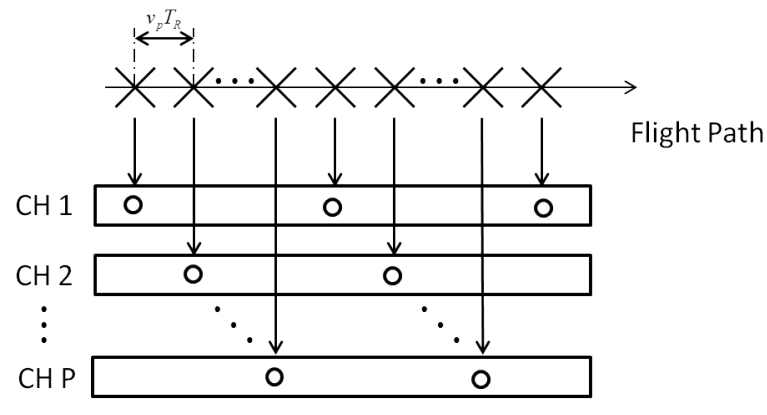

(b)

Figure 1. Acquisition geometry (a), Data rearranging (b)

\section{Signal Model}

Let consider the conventional SAR acquisition geometry in which a single channel radar mounted on an airborne platform illuminates a scene in which non-cooperative moving targets are present. This geometry is depicted in Fig.1 (a) in which the centre of the reference system $T_{\xi}$ is in the phase centre of the transmitter at $t=m T_{R}=0$. The reference system $T_{\xi}$ is oriented so that the axis $\xi_{2}$ is parallel to the radar LoS while $\xi_{1}$ is parallel to the flight path. The target motion can be expressed as the superimposition of the translational motion of the reference point, $R_{0}(m)$, and the rotational motion denoted by the rotation vector $\boldsymbol{\Omega}_{T}(m)$ applied to the reference point [7] [10]. The projection of $\boldsymbol{\Omega}_{T}(m)$ onto the plane orthogonal to the $\operatorname{LoS}$ is the effective rotation vector $\boldsymbol{\Omega}(m)$ and it is the component that produce radar-target aspect angle variation exploited to obtain cross-range resolution capability. The reference system $T_{x}$ has its origin on an arbitrary point on the target with the axis $x_{2}$ oriented along the LoS and the axis $x_{3}$ oriented along $\boldsymbol{\Omega}(m)$. The reference system $T_{y}$ is fixed on the target and coincides with $T_{x}$ for $m=0$. The angle $\alpha$ is the angle between $\xi_{3}$ and $x_{3}$.

The discrete slow time-frequency model of the received signal after matched filter is

$$
\begin{aligned}
S(n, m) & \doteq S\left(f_{0}+n \Delta f, m T_{R}\right) \\
& =S_{t}(n, m)+S_{c}(n, m)+N(n, m)
\end{aligned}
$$

where $S_{t}(n, m), S_{c}(n, m)$ and $N(n, m)$ denote the contributions due to the moving targets, static scene and the additive noise respectively. The indices $n=\left[-\frac{N}{2}, \ldots, \frac{N}{2}-1\right]$ and $m=\left[-\frac{M}{2}, \ldots, \frac{M}{2}-1\right]$ denote the frequency and pulse respectively and $\Delta f$ and $T_{R}=\frac{1}{P R F}$ denote the frequency sampling step and the Pulse Repetition Interval (PRI) respectively. It is well known from Fourier theory that the Doppler non-ambiguous region is determined by the $P R F$. Under the hypothesis that the PRF is higher than the 
Doppler occupancy of the SAR scene $\left(P R F>B_{D}\right)$ subsampling in the pulse domain can be applied without distorting the SAR image (only a reduction of the integration gain is introduced). This sub sampling allows the acquired data to be rearranged in such a way to emulate a multichannel SAR (MSAR) system as depicted in Fig.1. As shown in Fig.1 the sample acquired at the first pulse is considered as acquired by the first virtual channel, the second pulse by the second virtual channel and so on. The signal acquired by the $p^{\text {th }}$ virtual channel can then be expressed as

$$
\begin{aligned}
S_{p}\left(n, m^{\prime}\right) & \doteq S_{p}\left(f_{0}+n \Delta f, m^{\prime} T_{R}^{\prime}\right) \\
& =S\left(f_{0}+n \Delta f, m^{\prime} T_{R}^{\prime}+p T_{R}\right)
\end{aligned}
$$

where $p=[0, \ldots, P-1]$ is the virtual channel index, $m^{\prime}=\left[-\frac{M^{\prime}}{2}, \ldots, \frac{M^{\prime}}{2}-1\right]$ is the pulse index for the signal at each $p^{t h}$ channel. The number of pulses and the PRI for each channel are $M^{\prime}=\frac{M}{P}$ and $T_{R}^{\prime}=P \cdot T_{R}$ respectively. It is obvious that the non-ambiguous Doppler region for each channel is reduced with respect the one obtained processing the whole signal as $P R F^{\prime}=\frac{P R F}{P}$. It is quite obvious that for a fixed pulse index $m^{\prime}$ the signals acquired by the $P$ channels are not simultaneous as it would be in an actual multichannel SAR system. This effect should be taken into account in the signal modelling, especially in the statistical description of the clutter contribution.

\section{A. Target Component}

The model of the target contribution, $S_{t, p}\left(n, m^{\prime}\right)$, can be derived as in [5] under the assumptions that the straight iso-range approximation holds true and the virtual array size is much smaller than the radar target distance. The received signal from the generic virtual channel $p^{\text {th }}$ relative to the $k^{\text {th }}$ scatterer can be expressed as

$$
S_{t, p}\left(n, m^{\prime}\right)=\sigma_{k} e^{-j \frac{4 \pi\left(f_{0}+n \Delta f\right)}{c}\left[R_{0}^{(p)}\left(m^{\prime}\right)+\mathbf{y}^{(k)} \cdot \mathbf{i}_{L o S}^{(p)}\left(m^{\prime}\right)\right]}
$$

where $\sigma_{k}$ is the reflectivity of the $k^{t h}$ scatterer located at $\mathbf{y}^{(k)}, R_{0}^{(p)}\left(m^{\prime}\right)=R_{0}\left(m^{\prime} T_{R}^{\prime}+p T_{R}\right)$ is the distance between the $p^{t h}$ virtual receiver and the reference point on the target and $\mathbf{i}_{L o S_{y}}^{(p)}\left(m^{\prime}\right)=\mathbf{i}_{L o S_{y}}\left(m^{\prime} T_{R}^{\prime}+p T_{R}\right)$ is the LoS relative to the $p^{t h}$ virtual receiver expressed with respect to $T_{y}$. As shown in [5] [23] for small values of $T_{o b s}$ and, as a consequence, of a constant rotation vector during the observation time $\left(\boldsymbol{\Omega}_{T}(m) \approx \boldsymbol{\Omega}_{T}\right)$, the inner product in Eq.(3) can be expressed as follows

$$
\mathbf{y}^{(k)} \cdot \mathbf{i}_{L o S_{y}}^{(p)}\left(m^{\prime}\right)=K_{0, k}^{(p)}+K_{1, k}^{(p)} m^{\prime} T_{R}^{\prime}
$$

where

$$
\begin{aligned}
& K_{0, k}^{(p)}=y_{2}^{(k)}-\frac{v_{p} T_{R}}{R_{0}}\left[y_{1}^{(k)} p \cos (\alpha)-y_{3}^{(k)} p \sin (\alpha)\right] \\
& K_{1, k}^{(p)}=c_{2}^{(k)}-\frac{v_{p} T_{R}}{R_{0}}\left[c_{1}^{(k)} p \cos (\alpha)-c_{3}^{(k)} p \sin (\alpha)\right]
\end{aligned}
$$


and where $c_{1}^{(k)}, c_{2}^{(k)}=\Omega y_{1}^{(k)}$ and $c_{3}^{(k)}$ are the three components of the vector $\mathbf{c}^{(k)}=\boldsymbol{\Omega}_{T} \times \mathbf{y}^{(k)}$ as defined in [5].

The complex ISAR Point Spread Function (PSF) relative to a single scatterer placed in $\mathbf{y}^{(k)}$ can be obtained via Range Doppler (RD) processing after motion compensation [7],[10] and can be expressed as follows

$$
\begin{aligned}
& I^{(p)}(\tau, \nu)=B T_{o b s} \sigma_{k} e^{j 2 \pi f_{0}\left(\tau-\frac{2}{c} K_{0, k}^{(p)}\right)} \times \\
& \operatorname{sinc}\left[T_{\text {obs }}\left(\nu+\frac{2 f_{0}}{c} K_{1, k}^{(p)}\right)\right] \operatorname{sinc}\left[B\left(\tau-\frac{2}{c} K_{0, k}^{(p)}\right)\right]
\end{aligned}
$$

where $\tau$ and $\nu$ denote the delay time and the Doppler frequency respectively.

After the scaling operation (from $(\tau, \nu)$ to $\left(y_{1}, y_{2}\right)$ ) [7],[10] Eq.6 can be approximated as:

$$
\begin{aligned}
I^{(p)}\left(y_{1}, y_{2}\right)= & B T_{o b s} \sigma_{k} e^{j \frac{4 \pi f_{0}}{c}\left(y_{2}-K_{0, k}^{(p)}\right)} \times \\
& \operatorname{sinc}\left[T_{o b s}\left(y_{1}+y_{1}^{(k)}\right)\right] \operatorname{sinc}\left[B\left(y_{2}-y_{2}^{(k)}\right)\right]
\end{aligned}
$$

The Multichannel Range Doppler (M-RD) image is obtained by summing the $P$ complex images [5] and for $\alpha=0$ can be expressed as

$$
I_{M-R D}\left(y_{1}, y_{2}\right)=I^{(0)}\left(y_{1}, y_{2}\right) J\left(y_{1}\right)
$$

where the term $J\left(y_{1}\right)=\frac{\sin \left(\frac{2 \pi v_{p} T_{R} y_{1} P}{\lambda R_{0}}\right)}{\sin \left(\frac{2 \pi v_{R} T_{R} y_{1}}{\lambda R_{0}}\right)}$ takes into account the fact that the $P$ images sum in phase only in the focusing point and some distortion are introduced when the cross-range size of imaging area, $y_{1}$, or the virtual array size, $D=P v_{p} T_{R}$, are too large [5].

\section{B. Clutter Component}

In regard of the clutter contribution, $S_{c, p}\left(n, m^{\prime}\right)$ the model in [6][3] can be easily modified to take into account of the delayed response of each virtual channel. Details can be found in appendix A. In particular the clutter space-time covariance matrix, $\mathbf{R}$ can be expressed as

$$
\begin{aligned}
E & \left\{S_{c, p}(n, m) S_{c, q}^{*}(n, l)\right\} \\
& =P_{c} \rho_{s}\left[(l-m) v_{p} T_{R}^{\prime}+(p-q) v_{p} T_{R}\right] \\
& \times \rho_{t}\left[(l-m) T_{R}^{\prime}+(p-q) T_{R}\right]
\end{aligned}
$$

where $E\{\cdot\}$ denotes expectation, $(l, m)$ and $(p, q)$ denote the pulse and the virtual channel indexes respectively, $P_{c}$ is the clutter power and $\rho_{s}(\Delta \xi)=e^{-\frac{\Delta \xi^{2}}{2 \sigma_{s}^{2}}}$ and $\rho_{t}(\Delta t)=e^{-\frac{\Delta t^{2}}{2 \sigma_{t}^{2}}}$ are the spacial and temporal correlation coefficients respectively. 


\section{Remarks}

By observing Eq.(9) and Fig.1 a few considerations can be drawn. The baseline of the virtual M-SAR system, $d=v_{p} T_{R}$, and the virtual array size, $D=P v_{p} T_{R}$ depend on the radar $P R I$ and the platform velocity and can be imposed without taking into account the physical size of the antenna. Obviously, any variation of $v_{p}$ involves a change of the lower bound of the $P R F$ as discussed below (11) so the selection of $d$ and $D$ should be performed by taking into account this effect. Moreover, this allows the term $J\left(y_{1}\right)$ to be easily controlled. The non-simultaneous acquisition by the $P$ virtual channels is taken into account by the term $(p-q) T_{R}$ within the temporal correlation coefficient in Eq.(9). For stationary ground clutter this time decorrelation can be reasonably ignored so that the clutter statistical description is the same of an actual M-SAR. Obviously, the price to be paid is the reduction of the non-ambiguous Doppler region with respect the original single channel SAR data. In addition, in order to obtain the the virtual channels, the $P R F$ must be suitably chosen. In particular, an upper bound to the $P R F$ values is determined to avoid range ambiguities and is expressed as

$$
P R F \leq \frac{c}{2 D_{y_{2}}}
$$

where $D_{y_{2}}$ is the size of the illuminated swath along the range dimension. Obviously even Doppler ambiguities must be avoided so the following condition must be met

$$
P R F^{\prime}=\frac{P R F}{P} \geq B_{D}=\frac{2 D_{y_{1}} f_{0} v_{p}}{c R_{0}}
$$

where $D_{y_{1}}$ is the size of the illuminated area along the cross-range dimension.

\section{Clutter Suppression AND IMAGing}

The availability of virtual M-SAR allows for the Space Doppler Adaptive Processing (SDAP) to be applied. SDAP processing and its particularities have been presented in our previous works [4][5]. A brief review will be provided here for the sake of clarity. Only the sub-optimum approach is reviewed since this is the only one applicable in a realistic scenario as explained in [5].

The whole signal vector in the space Doppler domain is defined as

$$
\begin{aligned}
\tilde{\mathbf{S}}(n) & =\left[\tilde{\mathbf{S}}(n, 0), \tilde{\mathbf{S}}(n, 1), \ldots, \tilde{\mathbf{S}}\left(n, M^{\prime}-1\right)\right]^{T} \\
& \in C^{M^{\prime} P \times 1}
\end{aligned}
$$

where

$$
\begin{aligned}
\tilde{\mathbf{S}}\left(n, m_{\nu}\right) & =\frac{1}{P}\left[\tilde{S}_{1}\left(n, m_{\nu}\right), \tilde{S}_{2}\left(n, m_{\nu}\right), \ldots, \tilde{S}_{P}\left(n, m_{\nu}\right)\right]^{T} \\
& \in C^{P \times 1}
\end{aligned}
$$




$$
\tilde{S}_{p}\left(n, m_{\nu}\right)=D F T_{m^{\prime}}\left\{S_{p}\left(n, m^{\prime}\right)\right\}
$$

and $m_{\nu}$ is the Doppler frequency index. The whole reference vector in the space-Doppler domain is expressed as

$$
\begin{aligned}
& \tilde{\mathbf{G}}_{D}\left(n, m_{\nu}\right)= \\
& {\left[\tilde{\mathbf{S}}_{r e f}\left(n, m_{\nu}\right), \tilde{\mathbf{S}}_{r e f}\left(n, m_{\nu}-1\right), \ldots, \tilde{\mathbf{S}}_{r e f}\left(n, m_{\nu}-\left(M^{\prime}-1\right)\right)\right]^{T}} \\
& \in C^{M^{\prime} P \times 1}
\end{aligned}
$$

where

$$
\begin{aligned}
& \tilde{\mathbf{S}}_{r e f}\left(n, m_{\nu}\right)= \\
& \frac{1}{P}\left[\tilde{S}_{r e f, 1}\left(n, m_{\nu}\right), \tilde{S}_{r e f, 2}\left(n, m_{\nu}\right), \ldots, \tilde{S}_{r e f, P}\left(n, m_{\nu}\right)\right]^{T} \\
& \in C^{P \times 1}
\end{aligned}
$$

and

$$
\tilde{S}_{r e f, p}\left(n, m_{\nu}\right)=D F T_{m^{\prime}}\left\{S_{r e f, p}\left(n, m^{\prime}\right)\right\}
$$

Since $M^{\prime}$ can be large the sub-optimum implementation splits the $M^{\prime}$ Doppler bins into sub-blocks of length $L$ and carries out the optimum cancelling filtering in each block separately before coherently summing the outputs to give:

$$
u_{D, w}\left(n, m_{\nu}\right)=\sum_{i} u_{D, i}\left(n, m_{\nu}\right)
$$

where

$$
u_{D, i}\left(n, m_{\nu}\right)=\tilde{\mathbf{W}}_{D, i}^{H}\left(n, m_{\nu}\right) \tilde{\mathbf{S}}_{i}(n)
$$

in which $\tilde{\mathbf{W}}_{D, i}\left(n, m_{\nu}\right)$ is the weightvector with respect to the $i^{t h}$ block expressed as

$$
\tilde{\mathbf{W}}_{D, i}\left(n, m_{\nu}\right)=\hat{\mathbf{R}}_{D c, i}^{-1} \tilde{\mathbf{G}}_{D, i}\left(n, m_{\nu}\right)
$$

where $\tilde{\mathbf{G}}_{D, i}\left(n, m_{\nu}\right), \tilde{\mathbf{S}}_{i}(n)$ and $\hat{\mathbf{R}}_{D c, i}$ are the reference vector, the signal vector and the cross-power spectral matrix in the $i^{t h}$ block expressed as

$$
\tilde{\mathbf{G}}_{D, i}\left(n, m_{\nu}\right)=\left[\begin{array}{c}
\tilde{\mathbf{S}}_{r e f}\left(n, m_{\nu}-(i-1) L\right) \\
\tilde{\mathbf{S}}_{r e f}\left(n, m_{\nu}-((i-1) L+1)\right) \\
\tilde{\mathbf{S}}_{r e f}\left(n, m_{\nu}-((i-1) L+2)\right) \\
\vdots \\
\tilde{\mathbf{S}}_{r e f}\left(n, m_{\nu}-(i L-1)\right)
\end{array}\right] \in C^{L P \times 1}
$$




$$
\tilde{\mathbf{S}}_{i}(n)=\left[\begin{array}{c}
\tilde{\mathbf{S}}(n,(i-1) L) \\
\tilde{\mathbf{S}}(n,(i-1) L+1) \\
\tilde{\mathbf{S}}(n,(i-1) L+2) \\
\vdots \\
\tilde{\mathbf{S}}(n, i L-1)
\end{array}\right] \in C^{L P \times 1}
$$

and

$$
\hat{\mathbf{R}}_{D c, i}=\frac{1}{N_{r}} \sum_{n_{r}=0}^{N_{r}-1} \tilde{\mathbf{Z}}_{i}\left(n_{r}\right) \tilde{\mathbf{Z}}_{i}^{H}\left(n_{r}\right) \in C^{L P \times L P}
$$

where $\tilde{\mathbf{Z}}_{i}\left(n_{r}\right)$ denotes the target-free data in the $n_{r}$ range cell relative to the $i^{\text {th }}$ window. At the output the clutter suppressed ISAR image in which the defocused moving targets can be detected is obtained. At this stage ISAR processing should be applied to each detected target in order to obtain well focused high resolution ISAR images [3][5].

\section{Simulation Results}

In order to demonstrate the effectiveness of the proposed processing, a dataset simulated by Warsaw University of Technology, Institute of Electronic Systems, has been used. The terrain has been modelled by using a DEM with size $500 m \times 500 m$. Two moving targets are present in the scene. Specifically, a large trailer truck with velocity $15 \mathrm{~m} / \mathrm{s}$ along the range direction and a GAZ 66 military truck with velocity $30 \mathrm{~m} / \mathrm{s}$ along the range direction. A point-like slow moving target $(v=3 \mathrm{~m} / \mathrm{s})$ has been added to better demonstrate the detection capabilities. The main simulation parameters are summarized in Tab.I. The value of platform velocity is reasonable for an airborne SAR system so the PRF bounds are typical values when designing SAR systems. The high $P R F$ value respects the condition on the bounds and allows a 3 channels M-SAR system to be emulated. The results obtained by processing the single channel full data, i.e. $P=1$, are shown in Fig.2. As it can be noted the non-ambiguous Doppler region is larger than the Doppler occupancy of the SAR image (see Fig.2 (a)). As can be easily noted the two fast target are visible because they appear outside the static scene Doppler region. The slow moving target is not visible neither in the Range Doppler SAR image nor in the clutter suppressed SAR image (Fig.2 (b)) where the range dimension has been expanded to illustrate only the region under test. Since no spatial degrees of freedom (DoFs) are available there is no selectivity in the Diretion of Arrival $(D o A)$ dimension (Fig.2 (c)). The filter null, evaluated for $D o A=0$, is as large as the static scene Doppler occupancy leading to the cancellation of the slow moving target return (Fig.2 (d)). Fig.3 shows the results obtained by rearranging the acquired data in such a way to obtain 3 virtual channels, $P=3$. As is evident by 


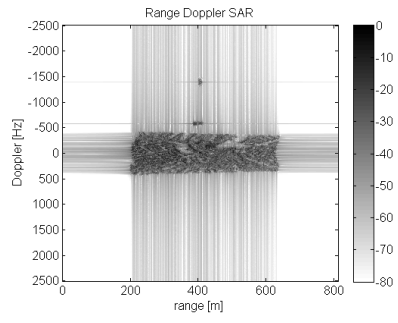

(a)

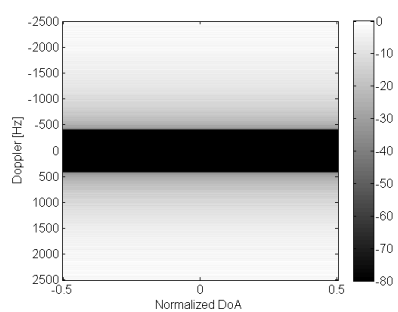

(c)

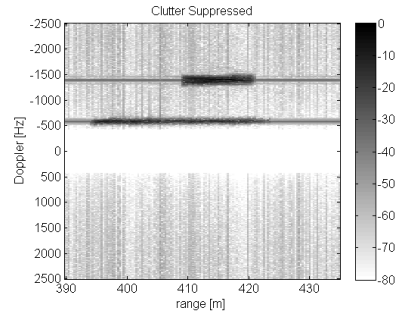

(b)

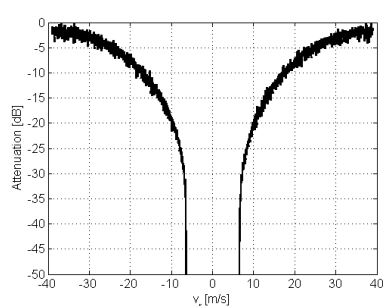

(d)

Figure 2. 1 actual channel: (a) Range Doppler SAR image, (b) Clutter Suppressed SAR image, (c) 2D Filter, (d) Filter Slice $D o A=0$

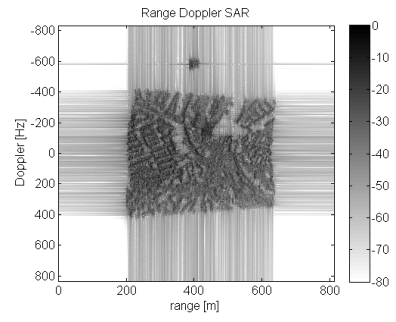

(a)

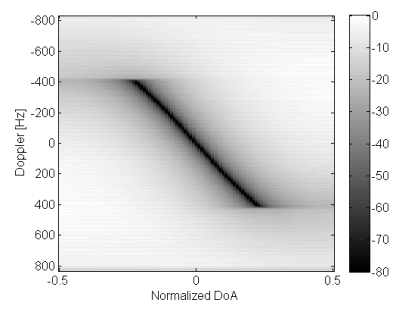

(c)

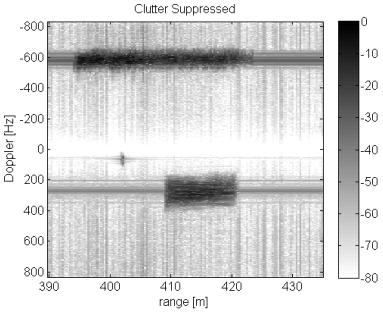

(b)

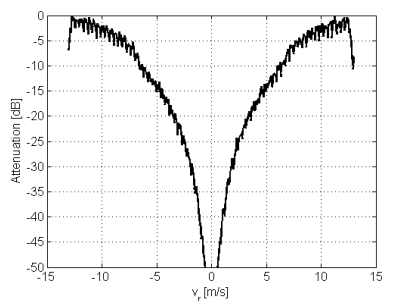

(d)

Figure 3. 3 virtual channels: (a) Range Doppler SAR image, (b) Clutter Suppressed SAR image, (c) 2D Filter, (d) Filter Slice $D o A=0$ 


\begin{tabular}{|c|c|}
\hline Carrier frequency $f_{0}$ & $9.6 \mathrm{GHz}$ \\
\hline PRF & $5 \mathrm{kHz}$ \\
\hline TX Bandwidth & $500 \mathrm{MHz}$ \\
\hline ADC Sampling frequency & $500 \mathrm{MHz}$ \\
\hline Platform Velocity & $50 \mathrm{~m} / \mathrm{s}$ \\
\hline Incident Angle & $55^{\circ}$ \\
\hline Antenna Beamwidth & $\theta_{e l}=20^{\circ}, \theta_{a z}=7.5^{\circ}$ \\
\hline Acquisition Time & $0.6 \mathrm{~s}$ \\
\hline \multicolumn{2}{|c|}{ Table I } \\
\hline
\end{tabular}

SIMULATION PARAMETERS

observing Fig.3 (a) the Doppler non-ambiguous region is reduced by the subsampling operation performed when producing the two additional virtual channels. Only one target out of three is visible in the RD SAR image formed by coherently summing the three SAR images formed from each of the virtual channels [5]. By applying the SDAP processing the clutter is well suppressed and all the three targets become visible in Fig.3 (b). The improvement in the detection capabilities are due to the availability of $P=3$ spatial DoFs that allows for a more selective filter to be synthesized as shown in Fig.3 (c) and (d) in which is evident that even slow moving targets can be preserved. It is worth highlighting that in Fig.3 (b) even the slow point-like moving target can be clearly seen. This is not possible without the synthesis of the 3 virtual channels. The comparison between the filter obtained with a system with 3 virtual channels and 3 actual channels can be performed exploiting the same simulated data set and is shown in Fig.4. As is evident the static clutter rejection capabilities with the two configuration are very similar. Obviously the synthesis of 3 virtual channels involves a reduction of the non-ambiguous Doppler region as explained above.

The results after the application of the ISAR processing to the targets detected in Fig.3 are shown in Fig.5. The improvement in the image focus is evident.

It is worth pointing out that, at the very end, the proposed processing performs a combination of weighted temporal samples. More specifically, in the case in which no virtual channels are considered (see Fig.2 (b)) all the available temporal samples are used to perform Doppler processing and there is no resolution along the $D o A$ dimension (see Fig.2 (c)). In the case in which virtual channels are synthesized (see Fig.3 (b)) the spatial diversity of bursts of temporal samples is used for applying spatial processing while only the remaining samples are used for the Doppler processing. In this second case the resolution capability in the DoA dimension is obtained (see Fig.2 (c)). Even if the weights in the two cases are the same, 


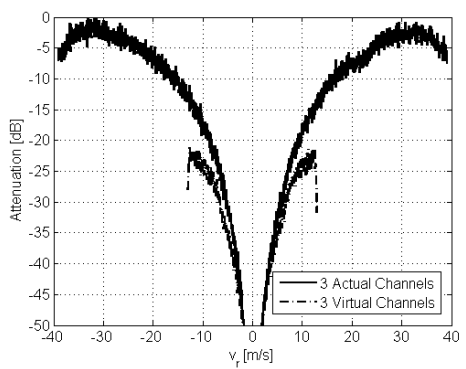

Figure 4. Filter Comparison

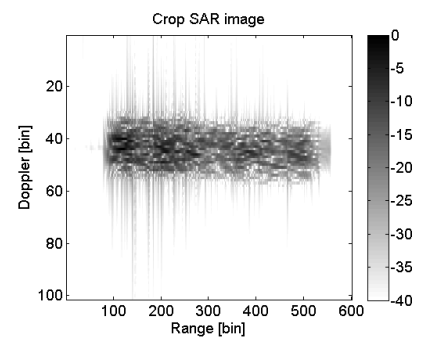

(a)

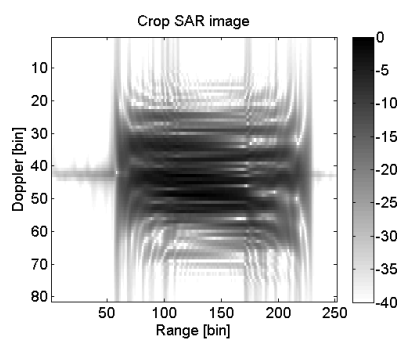

(c)

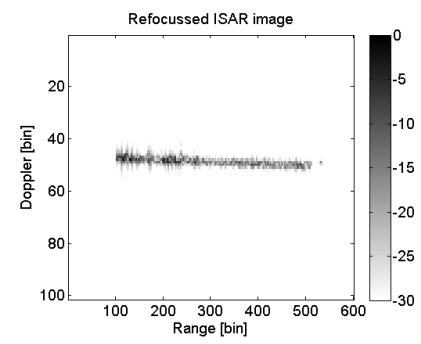

(b)

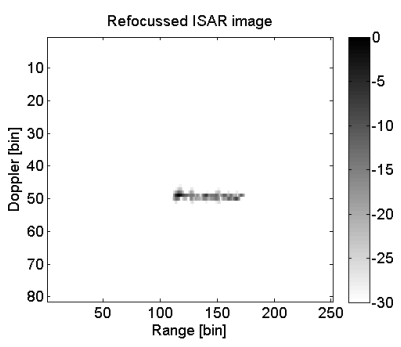

(d)

Figure 5. Target 1 SAR (a), Target 1 ISAR (b), Target 2 SAR (c), Target 2 ISAR (d)

the way in which the weighted samples are combined is intrinsically different and as a consequence the clutter rejection capability varies significantly. As stated above, the price to be paid is the reduction of the non-ambiguous region in the Doppler dimension and the scene must be stationary while the virtual array is formed. In other words, considering only one channel allows for only clutter temporal correlation to be taken into account while the synthesis of the virtual array allows for clutter spatial and temporal correlation to be jointly exploited in the clutter rejection processing. 


\section{EXPERIMENTAL RESULTS}

The proposed processing was tested on real data provided by Metasensing ${ }^{1}$ acquired using a twochannel SAR system with $P R F=5 \mathrm{kHz}$. A comparison with the results obtained with the synthesis of three virtual channels and the results obtained exploiting the two real channels was performed in order to demonstrate the effectiveness of the proposed architecture. The acquisition has been performed by flying over a highway in the proximity of Teuge airport. The highway was perpendicular to the flight trajectory. The acquisition parameters are summarized in Tab.II. The radar parameters are listed in Tab.III. Fig.6

\begin{tabular}{|c|c|}
\hline Platform & $\begin{array}{c}\text { 208 Grand Caravan from } \\
\text { Paracentrum Teuge (PH-SWP) }\end{array}$ \\
\hline Look angle & $55^{\circ}$ \\
\hline Flight Altitude & $1200 \mathrm{~m}$ \\
\hline Velocity & $180 \mathrm{~km} / \mathrm{h}$ \\
\hline Mean Range & $2 \mathrm{~km}$ \\
\hline Swath & $1 \mathrm{~km}$ \\
\hline
\end{tabular}

ACQUISITION PARAMETERS

\begin{tabular}{|c|c|}
\hline Center Frequency & $9.6 G H z$ \\
\hline TX Bandwidth & $120 M H z$ \\
\hline PRF & $5 k H z$ \\
\hline ADC sampling frequency & $25 \mathrm{MHz}$ \\
\hline \multicolumn{2}{|l|}{ TX: horn } \\
\hline Gain & $19.6 d B$ \\
\hline Antenna Aperture $(3 d B)$ & $\begin{array}{l}\text { elevation } 20^{\circ} \\
\text { azimuth } 20^{\circ}\end{array}$ \\
\hline \multicolumn{2}{|c|}{ RX: microstrip patch } \\
\hline Gain & $21.4 d B$ \\
\hline Antenna Aperture $(3 d B)$ & $\begin{array}{l}\text { elevation } 22^{\circ} \\
\text { azimuth } 7.5^{\circ}\end{array}$ \\
\hline Baseline & $15 \mathrm{~cm}$ \\
\hline
\end{tabular}

\footnotetext{
${ }^{1}$ www.metasensing.com
} 


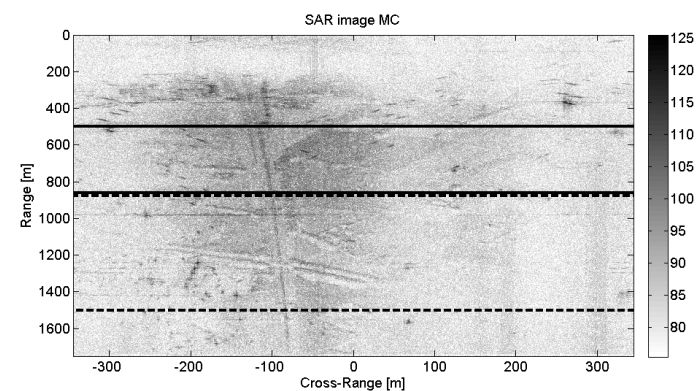

(a)

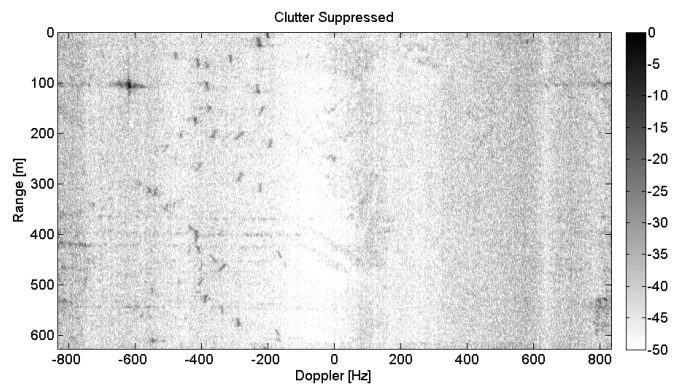

(c)

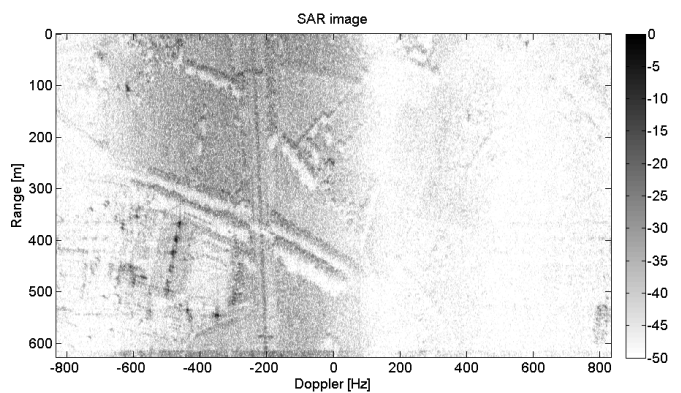

(b)

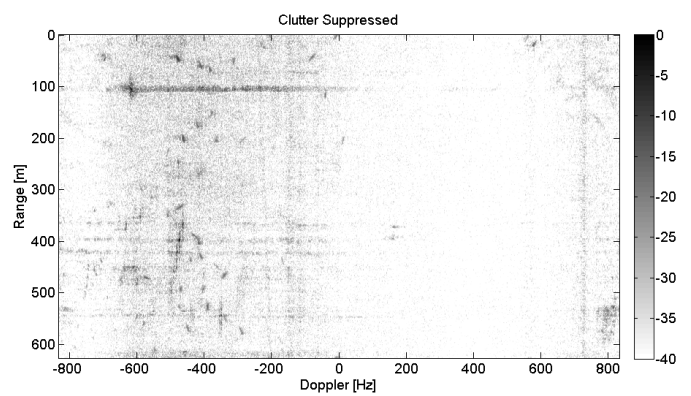

(d)

Figure 6. (a) SAR image with training region and region under test, (b) SAR image of the region under test, (c) Clutter Suppressed image with 3 virtual channels, (d) Clutter Suppressed image with 2 actual channels

(a) shows the SAR image obtained by applying the M-RD processing to the data acquired form the actual channel 1 rearranged in such a way to synthesizes $P=3$ virtual channels. The region under test and the training region are in the dashed and continuous box respectively. Fig.6 (b) and (c) show the M-RD SAR image and the clutter suppressed image of the region under test while Fig.6 (d) shows the clutter suppressed image obtained by considering the two actual channels presented in [5]. As is evident the clutter suppressed image obtained with three virtual channels is much better, from a visual point of view, than the image obtained with two actual channels. This is due to the fact that the availability of three virtual spatial DoFs allows for a better cancellation with respect the case of two actual DoFs. Moreover, the use of only one actual channel reduces the cross-channels calibration issues that may occur. In addition, as explained in [5], since the actual baseline is too long because of the physical size of the antennas, a baseline reduction process must be performed before applying the SDAP processing. This involves a decorrelation of the clutter that is larger with respect the case of three virtual channels. To better assess the performance in term of detection capabilities two sub-regions taken form Fig.6 are shown in Fig.7 and 8. Specifically the MR-SAR image obtained processing the three virtual channels are shown 
in Fig.7 (a) and Fig.8 (a). The clutter suppressed image obtained by exploiting the three virtual channels and the two actual channels are shown in (b) and (c) respectively while the ground truths associated with the two subregions are shown in Fig.7 (d) and Fig.8 (d). The Doppler frequency of every visible

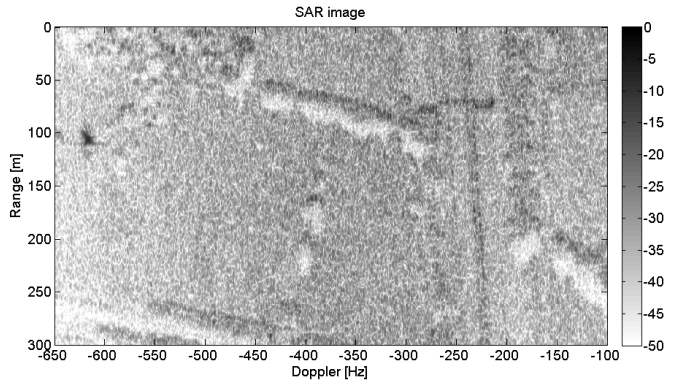

(a)

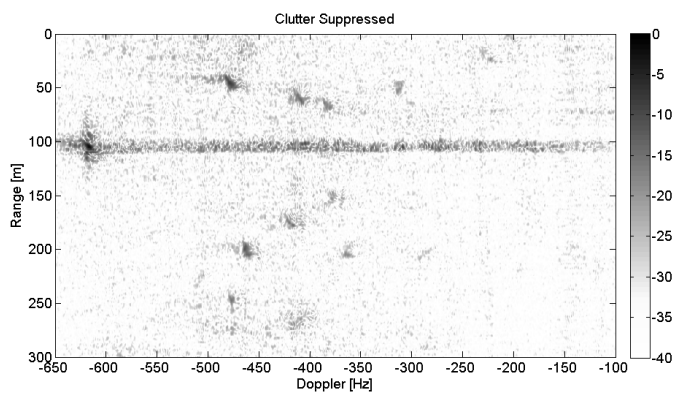

(c)

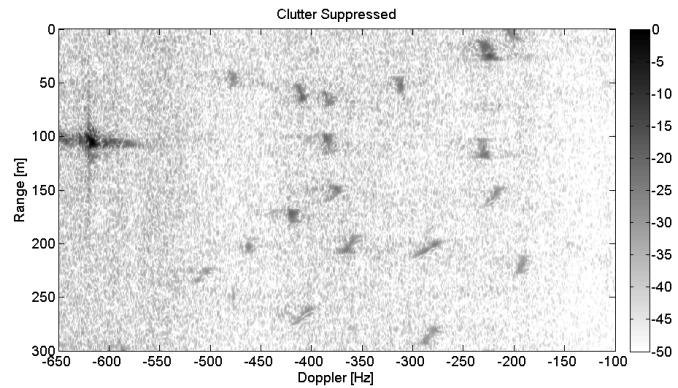

(b)

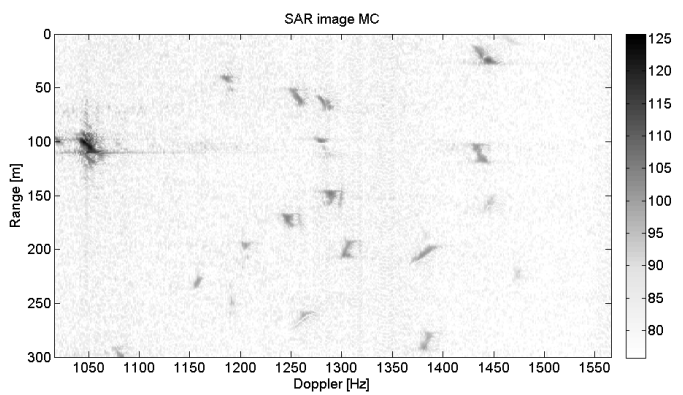

(d)

Figure 7. Sub-region 1 (a) SAR image with 3 virtual channels, (b) Clutter suppressed image with 3 virtual channels, (c) Clutter Suppressed image with 2 actual channels, (d) Pseudo ground truth

target in the ground truth images (Fig7 (d) and 8 (d)) are listed in Tab.IV-V as well as their Doppler frequency after subsampling $\left(f_{D}^{\prime}\right)$. The range coordinates of each target are also listed to easily locate the targets in Fig.7 and 8. The crosses indicate the missing targets in the clutter suppressed images obtained considering two actual channels and three virtual channels respectively. As can be easily noted, with three virtual channels, the number of missing targets is substantially reduced.

In order to demonstrate the imaging capabilities, the ISAR refocusing process has been applied to the targets detected after clutter cancellation. Results are shown in Fig.9 in which the SAR image and the refocused ISAR image are depicted. It should be noted that, the imaging capability is limited by the small system bandwidth. Nevertheless, as an indication of image quality the energy concentration can be considered and it is evident that this greatly increases after ISAR processing. In addition, the Image Contrast (IC) values before and after ISAR processing are shown in Tab.VI and VII for the targets detected 


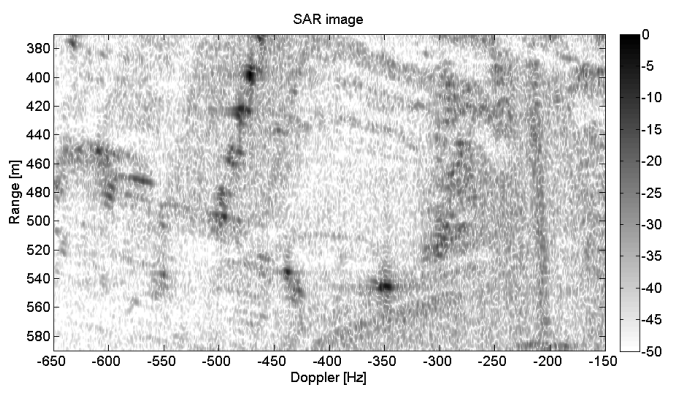

(a)

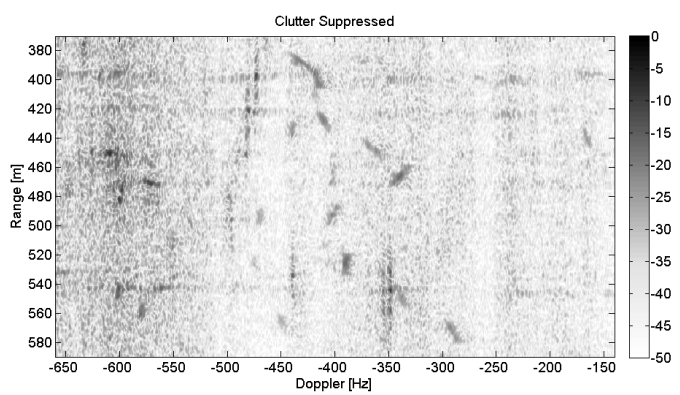

(c)

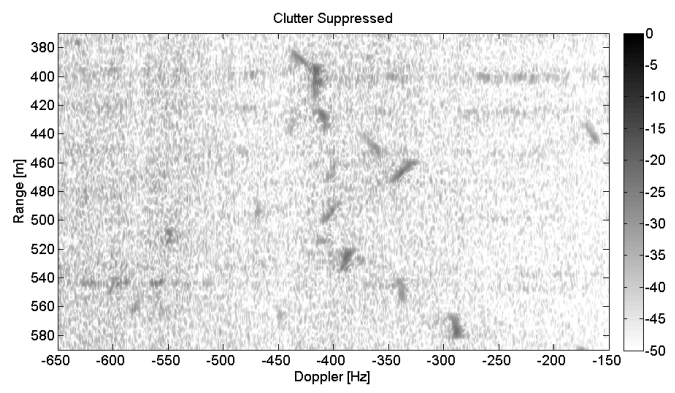

(b)

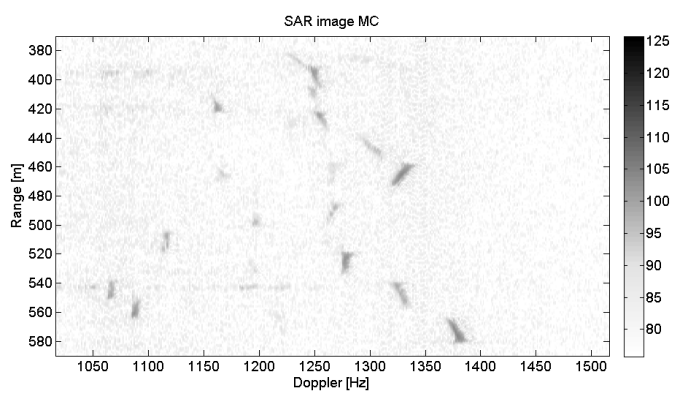

(d)

Figure 8. Sub-region 2 (a) SAR image with 3 virtual channels, (b) Clutter suppressed with 3 virtual channels, (c) Clutter Suppressed image with 2 actual channels, (d) Pseudo ground truth

in subregion 1 and 2, respectively. The blank boxes correspond to targets on which ISAR refocusing did not work. The improvement in image focus demonstrates the effectiveness of the proposed clutter suppression and moving target imaging processing when virtual channels are employed.

It is worth pointing out that the above mentioned analysis is performed by comparing results obtained by exploiting two actual channels and three virtual channels. This proves that the synthesis of virtual channels can easily overcome the drawback due to the hardware limitation. Nevertheless, the reader may argue that this is not a fair comparison since the number of spatial DoFs is different in the two cases. In order to prove the effectiveness of virtual multichannel with the same number of channels (real and virtual), the same analysis with two virtual channels are shown in Fig.10 .The value of sub-sampling factor is 3 (as in [5]) in order to obtain the same size of the non-ambiguous Doppler region. Specifically, Fig.10 (a) shows the M-RD SAR image, Fig.10 (b) shows the clutter suppressed image and Fig.10 (c) and (d) show the clutter suppressed images of the two considered subregion.

The improvement in the clutter suppression is evident by observing the above mentioned figures. In particular, in the results obtained with two actual channels more targets are cancelled and some clutter 


\begin{tabular}{|c|c|c|c|c|c|}
\hline Target N. & $\begin{array}{c}f_{D}[\mathrm{~Hz}] \\
\text { truth }\end{array}$ & $\begin{array}{c}f_{D}^{\prime}[\mathrm{Hz}] \\
\text { subsampled }\end{array}$ & $\begin{array}{c}\text { Range } \\
{[\mathrm{m}]}\end{array}$ & $\begin{array}{c}\text { Missing } \\
\text { targets } \\
2 \text { actual } \mathrm{Ch}\end{array}$ & $\begin{array}{c}\text { Missing } \\
\text { targets } \\
3 \text { virtual } \mathrm{Ch}\end{array}$ \\
\hline 1 & 1187 & -479 & 40 & & \\
\hline 2 & 1257 & -409 & 58 & & \\
\hline 3 & 1284 & -382 & 65 & & \\
\hline 4 & 1047 & -619 & 103 & & \\
\hline 5 & 1288 & -378 & 147 & & \\
\hline 6 & 1250 & -416 & 172 & & \\
\hline 7 & 1203 & -463 & 197 & & \\
\hline 8 & 1305 & -361 & 199 & & \\
\hline 9 & 1383 & -283 & 204 & $X$ & \\
\hline 10 & 1158 & -504 & 227 & $X$ & \\
\hline 11 & 1440 & -227 & 24 & $X$ & \\
\hline 12 & 1437 & -230 & 110 & $X$ & \\
\hline 13 & 1474 & -193 & 220 & $\mathrm{X}$ & \\
\hline 14 & 1384 & -283 & 282 & $X$ & \\
\hline
\end{tabular}

SUB-REGION 1: GROUND TRUTH COMPARISON

remaining can be clearly seen.

\section{CONCLUSION}

The applicability of SDAP-ISAR processing to obtain high resolution ISAR images of non-cooperative moving ground targets with a single channel SAR system has been demonstrated. Specifically, a method to obtain a virtual M-SAR system with an actual single channel SAR system has been presented. This can be achieved when the PRF is high enough to allow for subsampling in the slow time domain without introducing aliasing in the SAR scene return. Virtual M-SAR presents some advantages with respect to conventional M-SAR, such as the possibility to impose the baseline length without taking into account the physical size of the antennas but considering only the Doppler occupancy of the static scene and the use of a single receiver chain. Obviously this comes at the price of an higher $P R F$ that involves some 


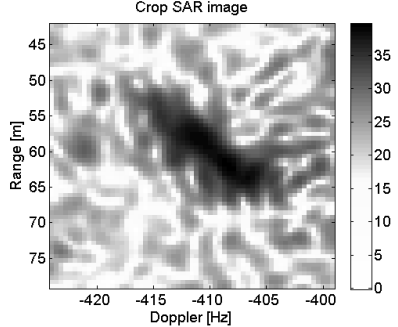

(a)

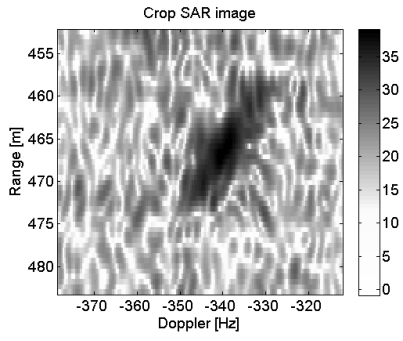

(c)

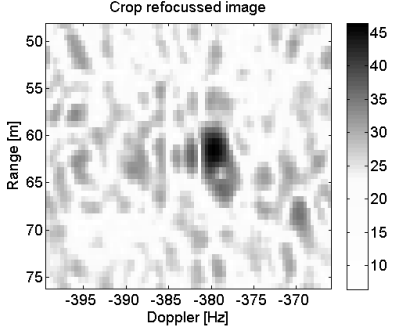

(b)

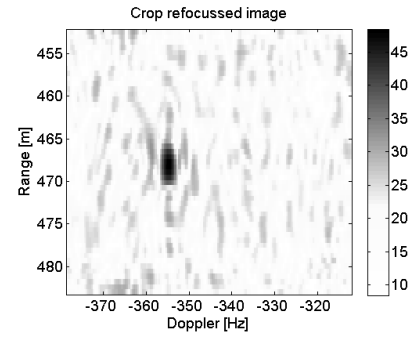

(d)

Figure 9. ISAR refocused (a) subregion 1 crop 2 SAR, (b) subregion 1 crop 2 ISAR, (c) subregion 2 crop 5 SAR, (d) subregion 1 crop 5 ISAR

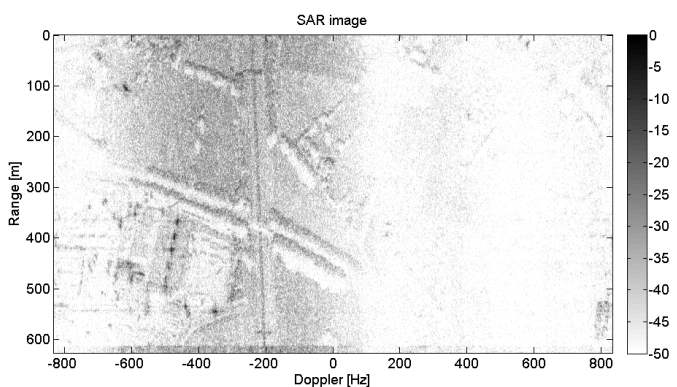

(a)

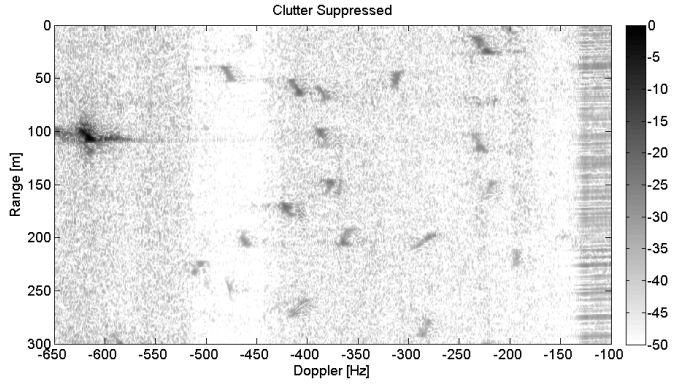

(c)

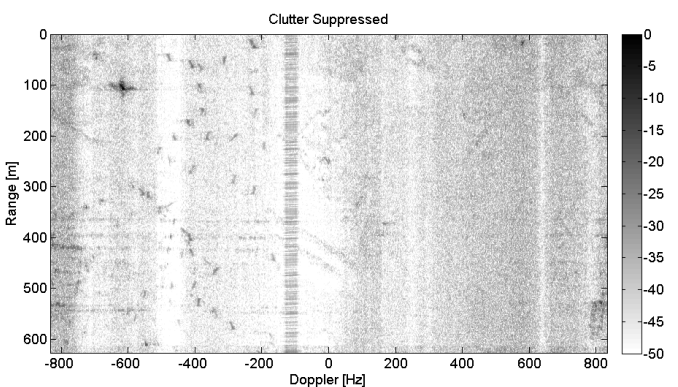

(b)

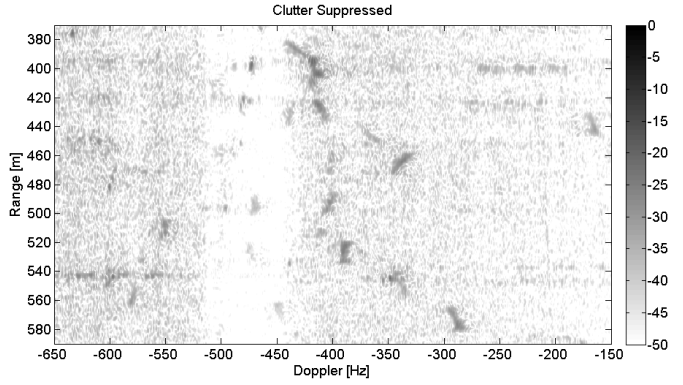

(d)

Figure 10. Results obtained with $P=2$ virtual channels: (a) M-RD SAR, (b) Clutter Suppressed, (c) Clutter Suppressed subregion 1, (d) Clutter Suppressed subregion 2 


\begin{tabular}{|c|c|c|c|c|c|}
\hline Target N. & $\begin{array}{c}f_{D}[\mathrm{~Hz}] \\
\text { truth }\end{array}$ & $\begin{array}{c}f_{D}^{\prime}[\mathrm{Hz}] \\
\text { subsampled }\end{array}$ & $\begin{array}{c}\text { Range } \\
{[\mathrm{m}]}\end{array}$ & $\begin{array}{c}\text { Missing } \\
\text { targets } \\
2 \text { actual } \mathrm{Ch}\end{array}$ & $\begin{array}{c}\text { Missing } \\
\text { targets } \\
3 \text { virtual } \mathrm{Ch}\end{array}$ \\
\hline 1 & 1255 & -412 & 395 & & \\
\hline 2 & 1165 & -502 & 419 & $X$ & $X$ \\
\hline 3 & 1257 & -410 & 427 & & \\
\hline 4 & 1301 & -366 & 445 & & \\
\hline 5 & 1332 & -355 & 465 & & \\
\hline 6 & 1268 & -399 & 489 & & \\
\hline 7 & 1198 & -469 & 498 & & \\
\hline 8 & 1117 & -550 & 510 & & \\
\hline 9 & 1281 & -386 & 524 & & \\
\hline 10 & 1667 & -600 & 543 & $X$ & \\
\hline 11 & 1329 & -338 & 546 & & \\
\hline 12 & 1090 & -577 & 558 & & \\
\hline 13 & 1379 & -288 & 573 & & \\
\hline
\end{tabular}

SUB-REGION 2: PSEUdO GROUND TRUTH COMPARISON

issues in the design process, and a larger amount of data to be acquired, stored and processed by a single receiver chain. The exploitation of a virtual M-SAR allows SDAP processing to be applied which leads to good clutter cancellation results even when compared to the case in which actual spatial DoFs are available. Real data results have been shown in order to further prove the effectiveness of the proposed architecture. A qualitative comparison with conventional processing has been performed.

\section{ACKNOWLEDGMENT}

The authors would like to thank Warsaw University of Technology, Institute of Electronic Systems and Metasensing for providing the simulated and the measured dataset respectively.

Part of this work has been carried out within the activities of the NATO-SET 196 on Multichannel/Multistatic Radar Imaging of Non-Cooperative Targets. 


\begin{tabular}{|c|c|c|}
\hline Target N. & IC SAR & IC ISAR \\
\hline \hline $\mathbf{1}$ & 1.519 & 5.649 \\
\hline $\mathbf{2}$ & 1.935 & 4.619 \\
\hline $\mathbf{3}$ & 1.766 & 4.238 \\
\hline $\mathbf{4}$ & 6.053 & 13.799 \\
\hline $\mathbf{5}$ & 1.655 & 4.685 \\
\hline $\mathbf{6}$ & 1.915 & 6.205 \\
\hline $\mathbf{7}$ & 1.96 & 2.853 \\
\hline $\mathbf{8}$ & 1.52 & 3.44 \\
\hline $\mathbf{9}$ & 9.968 & 3.41 \\
\hline $\mathbf{1 0}$ & 1.676 & 7.309 \\
\hline $\mathbf{1 1}$ & 2.573 & 6.699 \\
\hline $\mathbf{1 2}$ & 1.748 & 5.359 \\
\hline $\mathbf{1 3}$ & 1.622 & 7.589 \\
\hline $\mathbf{1 4}$ & 1.254 & 6.021 \\
\hline
\end{tabular}

SUB-REGION 1: IC VALUES BEFORE AND AFTER ISAR PROCESSING

\section{REFERENCES}

[1] BACCI, A. Optimal Space Time Adaptive Processing for Multichannel Inverse Synthetic Aperture Radar Imaging. PhD thesis, University of Pisa, Italy \& University of Adelaide, Australia, May 2014.

[2] Bacci, A., Gray, D., Martorella, M., And Berizzi, F. Joint STAP-ISAR for non-cooperative target imaging in strong clutter. In Radar Conference (RADAR), 2013 IEEE (2013), pp. 1-5.

[3] Bacci, A., Gray, D., Martorella, M., And Berizzi, F. Space-doppler processing for multichannel isar imaging of non-cooperative targets embedded in strong clutter. In Radar (Radar), 2013 International Conference on (2013), pp. 43-47.

[4] Bacci, A., Martorella, M., Gray, D., AND Berizzi, F. High resolution imaging of moving ground targets via clutter mitigation and isar processing. In Radar (Radar), 2014 International Conference on (2014).

[5] Bacci, A., Martorella, M., Gray, D., And Berizzi, F. Space-doppler adaptive processing for radar imaging of moving targets masked by ground clutter. IET Radar, Sonar Navigation (February 2015).

[6] Barbarossa, S., And Farina, A. Space-time-frequency processing of synthetic aperture radar signals. Aerospace and Electronic Systems, IEEE Transactions on 30, 2 (1994), 341-358.

[7] Berizzi, F., Mese, E., Diani, M., and Martorella, M. High-resolution isar imaging of maneuvering targets by means of the range instantaneous doppler technique: modeling and performance analysis. Image Processing, IEEE Transactions 


\begin{tabular}{|c|c|c|}
\hline Target N. & IC SAR & IC ISAR \\
\hline \hline $\mathbf{1}$ & 2.059 & 7.263 \\
\hline $\mathbf{3}$ & 1.7 & 5.158 \\
\hline $\mathbf{4}$ & & \\
\hline $\mathbf{5}$ & 1.789 & 7.795 \\
\hline $\mathbf{6}$ & 1.311 & 4.37 \\
\hline $\mathbf{7}$ & & \\
\hline $\mathbf{8}$ & 1.405 & 5.628 \\
\hline $\mathbf{9}$ & 1.405 & 5.628 \\
\hline $\mathbf{1 0}$ & & \\
\hline $\mathbf{1 1}$ & 0.434 & 3.253 \\
\hline $\mathbf{1 2}$ & & \\
\hline $\mathbf{1 3}$ & 2.215 & 7.976 \\
\hline & Table VII & \\
\hline
\end{tabular}

SUB-REGION 2: IC VALUES BEFORE AND AFTER ISAR PROCESSING

on 10, 12 (2001), 1880-1890.

[8] Carrara, W., Goodman, R., And Majewski, R. Spotlight Synthetic Aperture Radar: Signal Processing Algorithms. Artech House signal processing library. Artech House, Incorporated, 1995.

[9] Chapin, E., And Chen, C. Along-track interferometry for ground moving target indication. Aerospace and Electronic Systems Magazine, IEEE 23, 6 (2008), 19-24.

[10] Chen, V., And Martorella, M. Inverse Synthetic Aperture Radar Imaging; Principles, Algorithms and Applications. Institution of Engineering and Technology, 2014.

[11] Cohen, L. Time-frequency distributions-a review. Proceedings of the IEEE 77, 7 (1989), 941-981.

[12] D’Addio, E., Bisceglie, M. D., And Bottalico, S. Detection of moving objects with airborne $\{$ SAR $\}$. Signal Processing 36, 2 (1994), 149 - 162.

[13] Dickey, F.R., J., LAbitt, M., And Staudaher, F. Development of airborne moving target radar for long range surveillance. Aerospace and Electronic Systems, IEEE Transactions on 27, 6 (1991), 959-972.

[14] Ender, J. Space-time adaptive processing for synthetic aperture radar. In Space-Time Adaptive Processing (Ref. No. 1998/241), IEE Colloquium on (apr 1998), pp. 6/1 -618.

[15] ENDER, J. H. G. Space-time processing for multichannel synthetic aperture radar. Electronics Communication Engineering Journal 11, 1 (1999), 29-38.

[16] Gebert, N., Krieger, G., And Moreira, A. Digital beamforming on receive: Techniques and optimization strategies for high-resolution wide-swath sar imaging. Aerospace and Electronic Systems, IEEE Transactions on 45, 2 (April 2009), 
$564-592$.

[17] Gierull, C. Moving target detection by along-track sar interferometry. In DREO Technical Report (2002).

[18] Guerci, J. Space-time Adaptive Processing for Radar. Artech House radar library. Artech House, 2003.

[19] Klemm, R., of Engineering, I., And Technology. Principles of Space-Time Adaptive Processing, 3rd Edition. IET radar, sonar, navigation and avionics series. Institution of Engineering and Technology, 2006.

[20] Legg, J. A., Bolton, A. G., And Gray, D. A. Sar moving target detection using a non-uniform prf. In European SAR conference (1996), pp. 423-426.

[21] Lombardo, P. Estimation of target motion parameters from dual-channel sar echoes via time-frequency analysis. In Radar Conference, 1997., IEEE National (1997), pp. 13-18.

[22] Martorella, M., Giusti, E., Berizzi, F., Bacci, A., And Dalle Mese, E. Isar based techniques for refocusing non-cooperative targets in sar images. Radar, Sonar Navigation, IET 6, 5 (2012), 332-340.

[23] Martorella, M., Stagliano, D., Salvetti, F., And Battisti, N. 3d interferometric isar imaging of noncooperative targets. Aerospace and Electronic Systems, IEEE Transactions on 50, 4 (October 2014), 3102-3114.

[24] Pascazio, V., Schirinzi, G., And Farina, A. Moving target detection by along-track interferometry. In Geoscience and Remote Sensing Symposium, 2001. IGARSS '01. IEEE 2001 International (2001), vol. 7, pp. 3024-3026 vol.7.

[25] RANEY, R. Synthetic aperture imaging radar and moving targets. Aerospace and Electronic Systems, IEEE Transactions on AES-7, 3 (1971), 499-505.

[26] Rosenberg, L., AND Gray, D. Robust interference suppression for multichannel sar. In Signal Processing and Its Applications, 2005. Proceedings of the Eighth International Symposium on (28-31, 2005), vol. 2, pp. 883 - 886.

[27] Rosenberg, L., And Gray, D. Anti-jamming techniques for multichannel sar imaging. Radar, Sonar and Navigation, IEE Proceedings - 153, 3 (june 2006), $234-242$.

[28] Rosenberg, L., Trinkle, M., And Gray, D. Fast-time stap performance in pre and post range processing adaption as applied to multichannel sar. In Radar Symposium, 2006. IRS 2006. International (may 2006), pp. 1 -4.

[29] Skolnik, M. Radar Handbook, Third Edition. Electronics electrical engineering. McGraw-Hill Education, 2008.

[30] Ward, J. Space-Time Adaptive Processing for Airborne Radar. Massachusset Inst of Tech Lexinton Lincoln Lab, 1994.

[31] White, R. Change detection in sar imagery. In Synthetic Aperture Radar, IEE Colloquium on (1989), pp. 5/1-5/3.

\section{APPENDIX A}

\section{ClutTer MODELING}

The echo from a fixed scene can not be modelled in deterministic way. In this appendix a statistical model of the echo backscattered by the ground surface for the virtual multichannel SAR system is derived as in [6]. As known, the illuminated surface is subdivided into several adjacent cells of size depending on the SAR resolution. It is possible to characterize each resolution cell by the complex amplitude backscattering coefficient $A\left(\mathbf{y}, t_{1}\right)$; where $\mathbf{y}$ indicate the particular cell position and $t_{1}$ indicate the discrete slow-time pulse. Typically in monostatic case is assumed that the value of backscattering coefficient corresponding to a different cell positions is statistically independent, whereas the values corresponding to the same cell positions, observed at different time, is correlated by an amount depending 
on the time fluctuations of the surface. With this assumption, it is possible to define the correlation of the backscattering coefficient between values corresponding to different resolution cells at different time for the monostatic case as

$$
E\left\{A\left(\mathbf{y}, t_{1}\right) A^{*}\left(\mathbf{y}^{\prime}, t_{2}\right)\right\}=\sigma_{0} \delta_{0}\left(\mathbf{y}^{\prime}-\mathbf{y}\right) \rho_{t}\left(t_{2}-t_{1}\right)
$$

where $\delta_{0}$ is the Dirac pulse and $\rho_{t}$ is the time correlation coefficient. Considering the virtual channel $p$ at $l^{\text {th }}$ transmitted pulse and the virtual channel $q$ at the $m^{\text {th }}$ pulse. It is possible to express their slow-time position as

$$
\begin{gathered}
t_{1}=p T_{R}+l T_{R}^{\prime} \\
t_{2}=q T_{R}+m T_{R}^{\prime}
\end{gathered}
$$

Through the expression (25),(26), the backscattering correlation for the virtual case can be written as

$$
E\left\{A\left(\mathbf{y}, t_{1}\right) A^{*}\left(\mathbf{y}^{\prime}, t_{2}\right)\right\}=\sigma_{0} \delta_{0}\left(\mathbf{y}^{\prime}-\mathbf{y}\right) \rho_{t}\left[(p-q) T_{R}+(l-m) T_{R}^{\prime}\right]
$$

By considering the geometry onto the slant range plane shown in Fig.11, the overall echo, after range compression, backscattered by an elementary reflector located in $\mathbf{y}$ and received by the $p^{\text {th }}$ virtual element at the $l^{\text {th }}$ transmitted pulse can be expressed as

$$
r(\tau ; l, p)=K \int_{D_{\mathbf{y}}} \frac{G_{T}\left(\mathbf{R}_{0}^{(p)}(l), \mathbf{y}\right) G_{R}\left(\mathbf{R}_{0}^{(p)}(l), \mathbf{y}\right)}{\left|\mathbf{R}_{0}^{(p)}(l)-\mathbf{y}\right|^{2}} A(\mathbf{y}, l) \Phi_{s}\left(\tau-\frac{2}{c}\left|\mathbf{R}_{0}^{(p)}(l)-\mathbf{y}\right|\right) d \mathbf{y}
$$

where $R_{0}^{(p)}(l)$ identify the distance between the $p^{\text {th }}$ channel at the $l^{\text {th }}$ transmission pulse and the reference point on the target area, $G_{t x / r x}(\cdot)$ is the transmit/receive amplitude antenna radiation pattern that depending on the angle between the normal to the antenna and the Line of Side (LOS) while $K$ is a constant that depending on transmit power and $\Phi_{s}$ is the autocorrelation function of the transmitted signal. The triple integral in (28) is the projection onto the IPP, along $y_{3}$, and the integral over the flat illuminated surface, along $\left(y_{1}, y_{2}\right)$, indicated by $D$. Chirp signals are typically used as transmitted signal in order to obtain high range resolution, this fact involves narrow autocorrelation function that can be approximated by a function different from zero only within a window centred around $\tau=0$ whose with is proportional to the range resolution. Due to propriety of the signal autocorrelation function, the double integral in (28) can be well approximated by a line integral. Then if $\xi_{1}$ denote the line coordinate of the radar position, the coordinate of the virtual channel $p$ at the $l^{\text {th }}$ transmitted pulse can be expressed as

$$
\xi_{1, p}=p d+l v_{p} T_{R}^{\prime}
$$




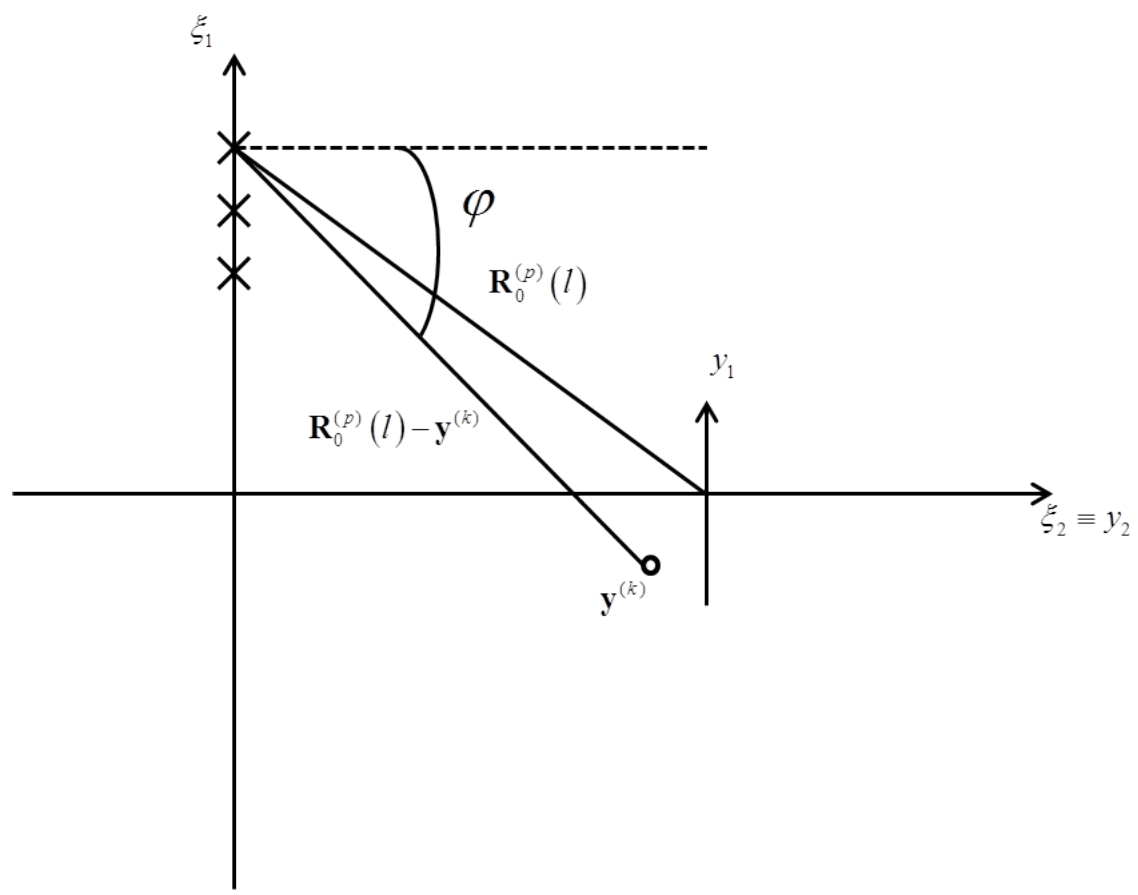

Figure 11. Geometry on slant-range plane

where $d=v_{p} T_{R}$ is the distance between adjacent virtual channel. Through these observations, the echo return received from $p^{\text {th }}$ channel from a particular range cell, expressed in (28), can be rewritten as

$$
r(l, p)=K^{\prime} \int_{D_{y_{1}}} G^{2}\left(\xi_{1, p}, y_{1}\right) A\left(y_{1}, l\right) d y_{1}
$$

where $y_{1}$ denotes the cell cross-range coordinate of $\mathbf{y}$. The distance from antenna element at particular transmitted pulse to the range cell, reported in the denominator of (28), can be assumed to be constant and included in the constant term $K^{\prime}$ while the antenna gains in transmission and reception can be consider proportional. As already mentioned, the amplitude antenna pattern depending on the angle $\varphi$ between the normal to the antenna and the LOS, then through simple geometrical relations it is possible to demonstrate the following expression

$$
\varphi=\operatorname{arctg}\left(\xi_{1, p}-y_{1}\right)
$$

therefore the function $G_{t x / r x}(\cdot)$ only depends on the difference $\xi_{1, p}-y_{1}$. As a result the integral in (30) can be finally rewritten as

$$
r(l, p)=K^{\prime} \int_{D_{y_{1}}} G^{2}\left(\xi_{1}-y_{1}\right) A\left(y_{1}, l\right) d y_{1}
$$


After derived the overall echo received from the generic virtual channel $p$ at the $l^{\text {th }}$ transmitted pulse, it is possible to evaluate the correlation between sample received from different virtual antenna elements at different transmitted pulses. Consider $p$ and $q$ as the virtual channels and $l$ and $m$ as the respectively transmitted pulses. It is possible to express the corresponding antenna coordinate respectively as

$$
\begin{gathered}
\xi_{1, p}=p d+l v_{p} T_{R}^{\prime} \\
\xi_{1, q}=q d+m v_{p} T_{R}^{\prime}
\end{gathered}
$$

After these considerations, the correlation can be defined as

$$
\begin{aligned}
& E\left\{r(l, p) r^{*}(m, q)\right\}= \\
& =E\left\{K^{\prime 2} \iint_{D_{y_{1}}, D_{y_{1}}} G^{2}\left(\xi_{1, p}-y_{1}\right) G^{2}\left(\xi_{1, q}-y_{1}^{\prime}\right) A\left(y_{1}, l\right) A^{*}\left(y_{1}^{\prime}, m\right) d y_{1} d y_{1}^{\prime}\right\}
\end{aligned}
$$

Using the relation reported in (27), it is possible to express (35) as

$$
\begin{aligned}
& E\left\{r(l, p) r^{*}(m, q)\right\}= \\
& =K^{\prime 2} \sigma_{0} \rho_{t}\left[(l-m) T_{R}^{\prime}+(p-q) T_{R}\right] \iint_{D_{y_{1}}, D_{y_{1}}} G^{2}\left(\xi_{1, p}-y_{1}\right) G^{2}\left(\xi_{1, q}-y_{1}^{\prime}\right) \delta_{0}\left(y_{1}^{\prime}-y_{1}\right) d y_{1} d y_{1}^{\prime}
\end{aligned}
$$

Define the $R_{G^{2}}(x)$ as the autocorrelation of the antenna radiation pattern, the expression in (36) can be rewritten as

$$
E\left\{r(l, p) r^{*}(m, q)\right\}=K^{\prime 2} \sigma_{0} \rho_{t}\left[(l-m) T_{R}^{\prime}+(p-q) T_{R}\right] R_{G^{2}}\left(\xi_{1, q}-\xi_{1, p}\right)
$$

Introducing the space correlation coefficient function $\rho_{s}(\xi)$, defined as the function $R_{G^{2}}(\xi)$ normalized to its maximum value, it is possible to obtain the finally expression of the correlation between the echo received by the $p^{\text {th }}$ virtual channel at the $l^{t h}$ transmitted pulse and the $q^{\text {th }}$ virtual channel at the $m^{\text {th }}$ pulse as

$$
E\left\{r(l, p) r^{*}(m, q)\right\}=P_{c} \rho_{t}\left[(l-m) T_{R}^{\prime}+(p-q) T_{R}\right] \rho_{s}\left[(p-q) v_{p} T_{R}+(l-m) v_{p} T_{R}^{\prime}\right]
$$

where $P_{c}$ is the power of the return echo. Its worth point out that the non-simultaneous acquisition due to the use of virtual array involves the presence of a temporal decorrelation term, taken into account by the expression $(p-q) T_{R}$, within the temporal correlation coefficient. Finally it should be noted that a good mathematical model for the time correlation function is the Gaussian, moreover by assuming a Gaussian antenna radiation pattern, the space correlation function is itself Gaussian. From these assumption, it is possible to express the space and time correlation as

$$
\rho_{s}(\Delta \xi)=e^{-\frac{\Delta \xi^{2}}{2 \sigma_{\xi}^{2}}}
$$




$$
\rho_{t}(\Delta t)=e^{-\frac{\Delta t^{2}}{2 \sigma_{t}^{2}}}
$$

where $\sigma_{s}$ and $\sigma_{t}$ the spatial and temporal standard deviations. 